\title{
Algorithm for calculating the rail-ground potential in heavy load conditions
}

\author{
Ivan Ignatenko ${ }^{1}$, Sergey Vlasenko ${ }^{1, *}$, Evgeniy Tryapkin ${ }^{1}$, and Vladimir Kovalev ${ }^{1}$ \\ ${ }^{1}$ Far Eastern State Transport University, 47, Seryshev Street, Khabarovsk, 680021, Russia
}

\begin{abstract}
Increased freight turnover on railway transport inevitably leads to increased traction current in DC and AC traction power supply systems. The increase in traction current is already causing problems related to the normal operation of the $25 \mathrm{kV}$ AC traction power supply systems. One of the adverse consequences of the increased traction currents is the increased rail-to-ground potential. This has already caused a number of accidents and related traffic interruptions on the Far Eastern Railway of Russia and other railway sections powered with alternating current. The study considers the problem of increased rail-to-ground potentials and provides basic formulae for calculating the wave parameters of the rail network and rail-to-ground potentials. Various methods are given for calculating rail-to-ground potentials for a $25 \mathrm{kV} \mathrm{AC}$ traction power supply system. Since in an alternating current system, expressions for calculating the potential are functions of a complex variable, the calculation of such expressions requires the use of special programs. Adaptation of existing methods to modern software and computing systems allows you to optimize and significantly speed up the process of calculating the "rail-to-ground" potentials, either considering the use of certain potential-reducing measures or not. A calculation method includes an algorithm developed for calculating the rail-to-ground potentials in the $25 \mathrm{kV} \mathrm{AC}$ traction power supply system for an inter-substation zone of any length with any number of electric locomotives within the zone.
\end{abstract}

\section{Introduction}

Methods for calculating rail-to-ground potentials are based on the electromagnetic field theory. Research papers present various approaches to determining the rail-to-ground potentials which differ in the proposed mathematical apparatus [1-4]. However, they share a common approach to considering the rail network as a line with distributed parameters [5].

\section{Materials and methods}

Figures and tables, as originals of good quality and well contrasted, are to be in their final form, ready for reproduction, pasted in the appropriate place in the text. Try to ensure that

\footnotetext{
*Corresponding author: vsa ens@mail.ru
} 
the size of the text in your figures is approximately the same size as the main text $(10$ point). Try to ensure that lines are no thinner than 0.25 point. We will use two methods to calculate the rail-to-ground potentials [6-8].

The mathematical model for calculating the rail-to-ground potentials of a reverse traction network is built on the following assumptions:

1. All rail networks of sections and stations of lengths $l_{i}$ form an infinite sequence of sections with uniform rail-to-ground transition resistance and wave parameters. The rail resistance is constant over the entire range of changes in the reverse traction current [9];

2. Reverse traction current is distributed evenly between the rails [10];

3. The complex potential "rail-to-ground" at any point of the traction rails is equal to the sum of the complex potentials from the currents flowing into the rails within the considered zone and depends only on the primary (first) harmonic of the currents [11].

The latter assumption allows applying the superposition method [12] when calculating the resulting value of the rail-to-ground potential created by several electric rolling stock (ERS). Thus, to find the resulting "rail-ground" potential at a point with the $x$ coordinate, it is enough to calculate the complex values of the potentials at this point created by each individual ERS, present them in algebraic form, sum the real and imaginary parts, and then calculate the module of the resulting value [13].

The calculated "instantaneous" scheme with two substations, sections with different $r_{r}$ and n-loads is shown in Fig. 1.

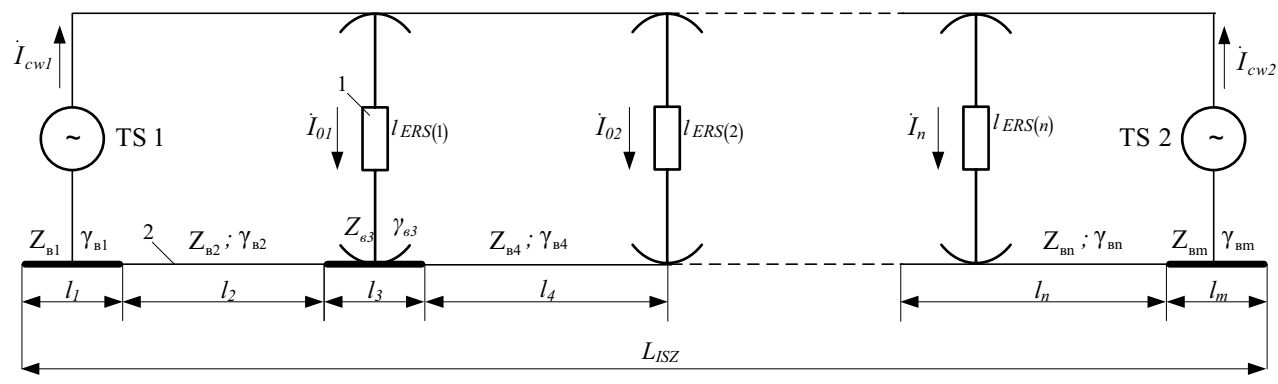

Fig. 1. Scheme for calculating rail potentials with two-way power supply and an arbitrary number of loads: 1 - load (ERS); 2 - rails; $I_{01} \ldots I_{n}$ - currents of ERS, A; $1_{\text {ERS }(1) \ldots l_{E R S(n)}}$ - distances of the i-th ERS from traction substation (TS) $1, \mathrm{~km} ; l_{1} \ldots l_{m}$ - length of sections with uniform resistance of $r_{c}$, $\mathrm{km}$; LISZ - length of the inter-substation zone (ISZ), $\mathrm{km}$.

In accordance with the method proposed in [14], the rail-to-ground potential created by the $\mathrm{i}$-th ERS at a point with the $x$ coordinate located to the left or right of the i-th ERS is calculated using expressions (1) and (2):

$$
\begin{aligned}
& \dot{\varphi}_{r-g(l) i}(x)=\frac{1}{2} \cdot \underline{z}_{b} \cdot(1-\mu) \cdot \dot{I}_{0 i} \cdot\left(\mathrm{e}^{-\dot{\gamma} \cdot x}-\left(1-\frac{l_{E R S(i)}}{L_{I S Z}}\right) \cdot \mathrm{e}^{-\dot{\gamma} \cdot\left(l_{\text {Inc }}(i)-x\right)}-\frac{l_{i}}{L_{\text {Mn } 3}} \cdot \mathrm{e}^{-\dot{\gamma} \cdot\left(L_{I S Z}-l_{E R S(i)}+x\right)}\right) \\
& \dot{\varphi}_{r-g(r) i}(x)=\frac{1}{2} \cdot \underline{z}_{6} \cdot(1-\mu) \cdot \dot{I_{0 i}}\left(\mathrm{e}^{-\dot{\gamma} \cdot x}-\left(1-\frac{l_{E R S C(i)}}{L_{I S Z}}\right) \cdot \mathrm{e}^{-\dot{\gamma} \cdot\left(l_{E R S(i)}+x\right)}-\frac{l_{i}}{L_{I S Z}} \cdot \mathrm{e}^{-\dot{\gamma} \cdot\left(L_{I S Z}-l_{E R S(i)}-x\right)}\right) .
\end{aligned}
$$

where $\mu$ is the relative value of the mutual induction resistance between the catenary system and the rail [15]. This coefficient is calculated using the formula (3): 


$$
\mu=\frac{\underline{z}_{c r}}{\underline{z}_{r}}
$$

where $\underline{z}_{c r}$ is the linear resistance of mutual induction between the catenary system and the rails, Ohms $/ \mathrm{km}$.

The mutual induction resistance of two conductors is calculated as the product of the mutual inductance of these conductors $M$ by the angular frequency of the current $\omega$ [16].

The mutual inductance between two infinitely long wires located above the earth's surface is calculated using the formula of F. Pollacek [17]:

$$
M \approx\left[2 \cdot \ln \left(\frac{2}{\alpha \cdot \gamma \cdot|k|}\right)+1-j \cdot \frac{\pi}{2}-j \cdot \frac{4 \cdot k}{3} \cdot\left(h_{1}+h_{2}\right)\right] \cdot 10^{-4},
$$

where $\alpha$ is the distance between the wires, $\mathrm{cm} ; \mathrm{h}_{1}$ and $\mathrm{h}_{2}$ are the heights of the wires above the ground, $\mathrm{cm} ; \gamma$ is the Euler constant $(\gamma=1,7811) ; k$ is a physical parameter that characterizes the electromagnetic properties of the system [18].

The parameter $\kappa$ is calculated using the formula (5):

$$
k=\sqrt{-4 \cdot \pi \cdot \omega \cdot \sigma \cdot j},
$$

where $\sigma$ is the specific conductivity of the earth, CGS units $\mu_{0}$ (1 unit). SGS $\mu_{0}=10^{11}$ $\mathrm{Cm} / \mathrm{m})$.

In accordance with the recommendations of [19], the last monomial of expression (4) can be ignored due to its smallness. Finally, the formula for calculating the mutual induction resistance is written as:

$$
\underline{z}_{M}=\omega \cdot\left(2 \cdot \ln \left(\frac{2}{\alpha \cdot \gamma \cdot|k|}\right)+1-j \cdot \frac{\pi}{2}\right) \cdot 10^{-4} .
$$

The scheme for calculating the mutual induction resistance between the catenary system and the rails for a one-track section is shown in Fig. 2.

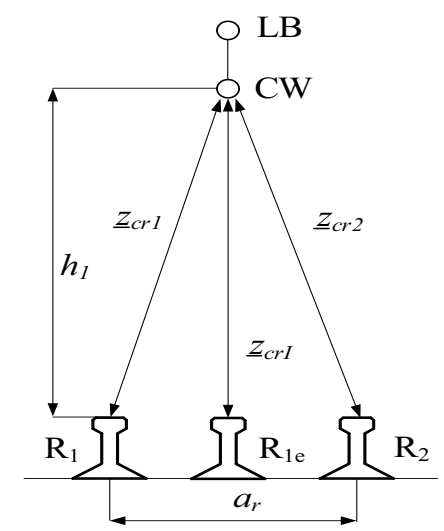

Fig. 2. Scheme for calculating the mutual induction resistance of the catenary system and rails of a one-track section: $\mathrm{R}_{1}$ and $\mathrm{R}_{2}$ are rails, $a_{r}$ is a track width, $\mathrm{R}_{1 \mathrm{e}}$ is an equivalent rail, $\mathrm{LB}$ is a loadbearing cable, $\mathrm{CW}$ is a contact wire. 
Taking $\alpha=h_{1}=h_{c w}$, the mutual induction resistance between the catenary system and the rails of a one-track section (for $\omega=314 \mathrm{rad} / \mathrm{s}$ ) [19] can be calculated using the following formula:

$$
\underline{z}_{c r I}=0,0628 \cdot \ln \left(\frac{5652,908}{h_{c w} \cdot \sqrt{\sigma}}\right)+0,0314-0,0493 \cdot j
$$

where $h_{\mathrm{cw}}$ is the section-average height of the contact wire suspension relative to the railhead level, $\mathrm{cm}$.

The scheme for calculating the mutual induction resistance between the catenary system and the rails for a two-track section is shown in Fig. 3.

In accordance with [20], the resistance of mutual induction between the catenary system and the rails of a two-track section is determined by the formula:

$$
\underline{z}_{c r I I}=0,5 \cdot\left(\underline{z}_{c r I}+\underline{z}_{c r I^{\prime}}\right)
$$

where $\underline{z}_{\text {crl' }}$ is the mutual induction resistance between the catenary system of the 1 st track and the rails of the $2 \mathrm{nd}$ track, Ohms $/ \mathrm{km}$.

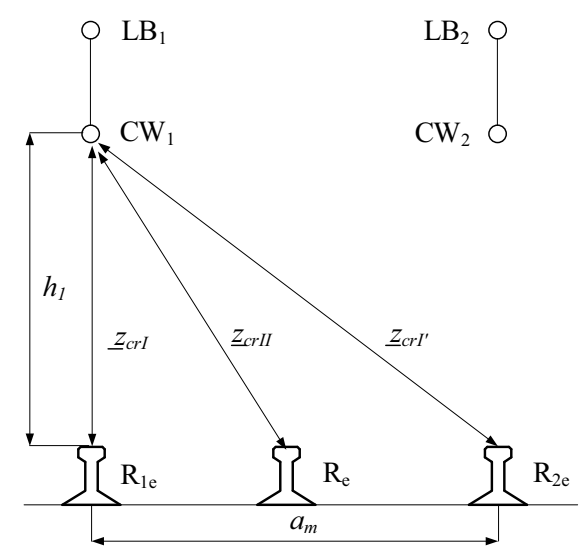

Fig. 3. Scheme for calculating the mutual induction resistance of the catenary system and rails of a two-track section: $\mathrm{R}_{1}$ and $\mathrm{R}_{2}$ are rails, $a_{m}$ is a track spacing, $\mathrm{R}_{1 \mathrm{e}}$ is an equivalent rail of the 1 st track, $\mathrm{R}_{2 \mathrm{e}}$ is an equivalent rail of the second track, $\mathrm{R}_{\mathrm{e}}$ is an equivalent rail of a two-track section.

Then, to determine $\underline{z}_{c r I^{\prime}}$ by the formula (6), we take $\alpha=\sqrt{\left(h_{1}\right)^{2}+\left(a_{r}+a_{m}\right)^{2}}$. Finally, on substituting $\underline{z}_{c r I}$ and $\underline{z}_{c r I}$ in the formula (8) and performing transformations, we obtain the formula:

$$
\underline{z}_{c r I I}=0,0314 \cdot \ln \left(\frac{5652,908}{h_{c w} \cdot \sqrt{\sigma}}\right)+0,0314+0,0314 \cdot \ln \left(\frac{5652,908}{\sqrt{\sigma} \cdot \sqrt{315844+h_{c w}^{2}}}\right)-0,0493 \cdot j,
$$

where $h_{c w}$ is the same as in formula (7).

The formula (9) is obtained based on the provisions that, in accordance with [21], the distance between the axes of railway tracks on running lines of two-track railway lines on straight sections is $410 \mathrm{~cm}$. 
In accordance with [22], the equivalent (linear) resistance of the rails of a one-track section can be calculated using the expression:

$$
\underline{z}_{r I}=\frac{r}{2}+0,05-j \cdot 0,144 \cdot\left(1,53-5,2 \cdot \frac{r}{2}+\lg \left(\sqrt{R \cdot a_{r}} \cdot \sqrt{\sigma}\right)\right),
$$

where $r_{r a}$ is the active linear resistance of the rail, Ohms $/ \mathrm{km} ; R$ is the equivalent radius of a circular cylinder whose area is equal to the cross-sectional area of the rail, $\mathrm{cm}$.

In accordance with [4], the equivalent (linear) resistance of the rails of a two-track section can be calculated using the expression:

$$
\underline{z}_{r I I}=\frac{r a}{4}+0,05-j \cdot 0,144 \cdot\left(1,53-5,2 \cdot \frac{r}{4}+\lg \left(\sqrt[4]{R \cdot a_{r} \cdot a_{m}^{2}} \cdot \sqrt{\sigma}\right)\right) .
$$

In [5], the scheme shown in Fig. 4 is considered as a calculation scheme:

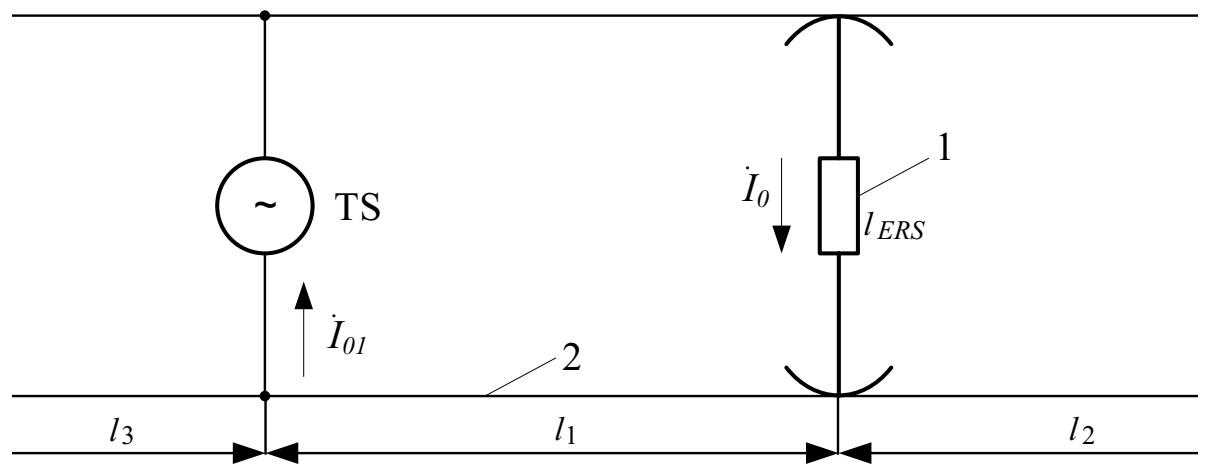

Fig. 4. Calculation scheme of rail-to-ground potentials: 1 - load (ERS); 2 - rails; $I_{0}$ - electric locomotive current, A; I01- reverse current feeder current, A.

To calculate the rail-to-ground potential, the following formula is proposed (12):

$$
\dot{\varphi}_{r-g}(x)=\underline{A}_{1} \cdot e^{-\dot{\gamma} \cdot x}+\underline{A}_{2} \cdot e^{\dot{\gamma} \cdot x},
$$

where $\underline{A}_{1}$ and $\underline{A}_{2}$ are coefficients.

Coefficients $\underline{A}_{1}$ and $\underline{A}_{2}$ are calculated using formulas (13) and (14):

$$
\underline{A}_{1}=-\frac{\dot{\varphi}_{0}-\dot{\varphi}_{01} \cdot e^{\dot{\gamma} \cdot l_{1}}}{2 \cdot \operatorname{sh}\left(\dot{\gamma} \cdot l_{1}\right)},
$$




$$
\underline{A}_{2}=-\frac{\dot{\varphi}_{0}-\dot{\varphi}_{01} \cdot e^{-\dot{\gamma} \cdot l_{1}}}{2 \cdot \operatorname{sh}\left(\dot{\gamma} \cdot l_{1}\right)},
$$

where $\dot{\varphi}_{0}$ is the potential of the reverse current circuit in the location point of the electric locomotive, V; $\dot{\varphi}_{01}$ is the potential of the reverse current circuit in the point of connection of the reverse current feeder, $\mathrm{V}$.

The potentials of the reverse current circuit at the location point of the electric locomotive and at the point where the reverse current feeder is connected are calculated using the formulas (15) and (16):

$$
\begin{aligned}
& \dot{\varphi}_{0}=\underline{z}_{B} \cdot(1-\mu) \cdot \dot{I}_{0} \cdot \underline{K}_{0}, \\
& \dot{\varphi}_{01}=-\underline{z}_{B} \cdot(1-\mu) \cdot \dot{I}_{o m} \cdot \underline{K}_{01},
\end{aligned}
$$

where $\underline{K}_{01}$ and $\underline{K}_{0}$ are coefficients; $\dot{I}_{\text {om }}$ is current in the reverse traction current feeder, A.

Formulas for calculating the coefficients $\underline{K}_{0}$ and $\underline{K}_{01}$. are presented in table 1 .

Table 1. Formulas for calculating coefficients $\underline{K}_{0}$ and $\underline{K}_{01}$.

\begin{tabular}{|c|c|c|c|}
\hline \multicolumn{2}{|c|}{ Lengths of sections } & \multicolumn{2}{|c|}{ Coefficient } \\
\hline$l_{1}$ & $l_{2}$ & $\underline{K}_{0}$ & $\underline{K}_{01}$ \\
\hline 0 & 0 & $0,5 \cdot \operatorname{th}\left(0,5 \cdot \dot{\gamma} \cdot l_{1}\right)$ & $0,5 \cdot \operatorname{th}\left(0,5 \cdot \dot{\gamma} \cdot l_{1}\right)$ \\
\hline$\infty$ & 0 & $0,5 \cdot\left(1-e^{-\dot{\gamma} \cdot l_{1}}\right)$ & $0,25 \cdot\left(1-e^{-\dot{\gamma} \cdot l_{1}}\right)^{2}$ \\
\hline$\infty$ & $\infty$ & $0,25 \cdot\left(1-e^{-\dot{\gamma} \cdot l_{1}}\right)$ & $0,25 \cdot\left(1-e^{-\dot{\gamma} \cdot l_{1}}\right)$ \\
\hline
\end{tabular}

Formulas (12)-(16), when taking $l_{1}=l_{E R S(i)}$, and for selecting coefficients in table 2.1 $l_{1}=\infty$ and $l_{2}=l_{3}=0$ allow calculating the rail-to-ground potentials in the section from the TS to the electric locomotive, provided that in formula (2.12) $\mathrm{x} \in\left[\begin{array}{ll}0 \ldots & 1_{1}\end{array}\right]$.

The calculation of the rail-to-ground potentials for a circuit with two substations using the formulas $(2.12)-(2.16)$ is proposed as follows:

Calculating the current in the reverse traction current feeder for TS 1 and TS 2 using the formulas (2.17) and (2.18).

For TS 1 


$$
\dot{I}_{01}=\dot{I}_{0} \cdot \frac{\left(L_{I S Z}-l_{1}\right)}{L_{I S Z}}
$$

For TS 2

$$
\dot{I}_{02}=\dot{I}_{0} \cdot \frac{l_{1}}{L_{I S Z}} .
$$

For the section "TS1 $-I_{E R S(i)}$ ", when selecting the coefficients in table $1_{1} l_{1}=\infty$ and $l_{2}=l_{3}=0$ are taken.

Making a calculation using the formulas (12)-(16) and taking $l_{1}=l_{E R S(i)}, x \in\left[\begin{array}{ll}0 \ldots & l_{1}\end{array}\right]$, and use $\dot{I}_{01}$ calculated by (17) in (16) as the current of the reverse current feeder.

For the section "IERS(i) - TS2", when selecting the coefficients in table $1_{1} l_{1}=\infty$ and $l_{2}=l_{3}=0$ are taken.

Making a calculation using the formulas (12)-(16) and taking $l_{1}=L_{I S Z}-l_{E R S(i)}, x \in\left[\left(\begin{array}{ll}L & I S Z\end{array}-l_{E R S(i)}\right) \ldots 0\right]$, and use $\dot{\mathrm{I}}_{02}$ calculated by (18) as the current of the reverse current feeder.

In both methods, the equations for calculating rail-to-ground potentials are functions of a complex variable. The calculation of these functions without the use of special software on the computer is very complicated and time consuming [23]. To improve the speed and accuracy of calculations, we consider it appropriate to adapt these equations, as well as the equations for calculating the values that characterize the traction power supply systems parameters to the software complex PascalABC.NET, which allows implementing mathematical models of physical processes using the classic Pascal programming language.

\section{Results}

Based on the obtained calculation method, we will create an adapted algorithm for the railto-ground potential calculation program (Fig. 5 - 8). 


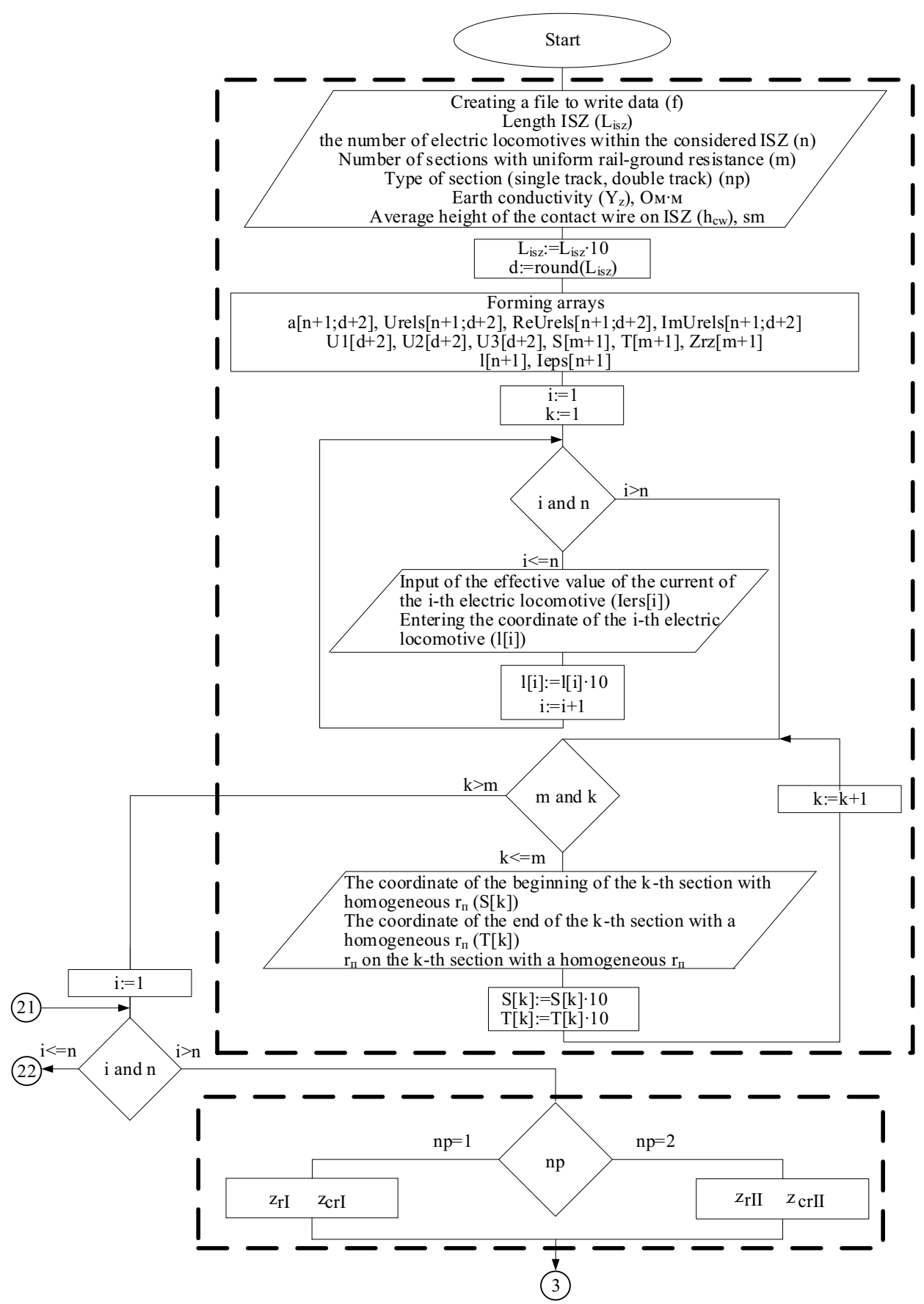

Fig. 5. Fragment №1 of the program operation algorithm. 
(22)

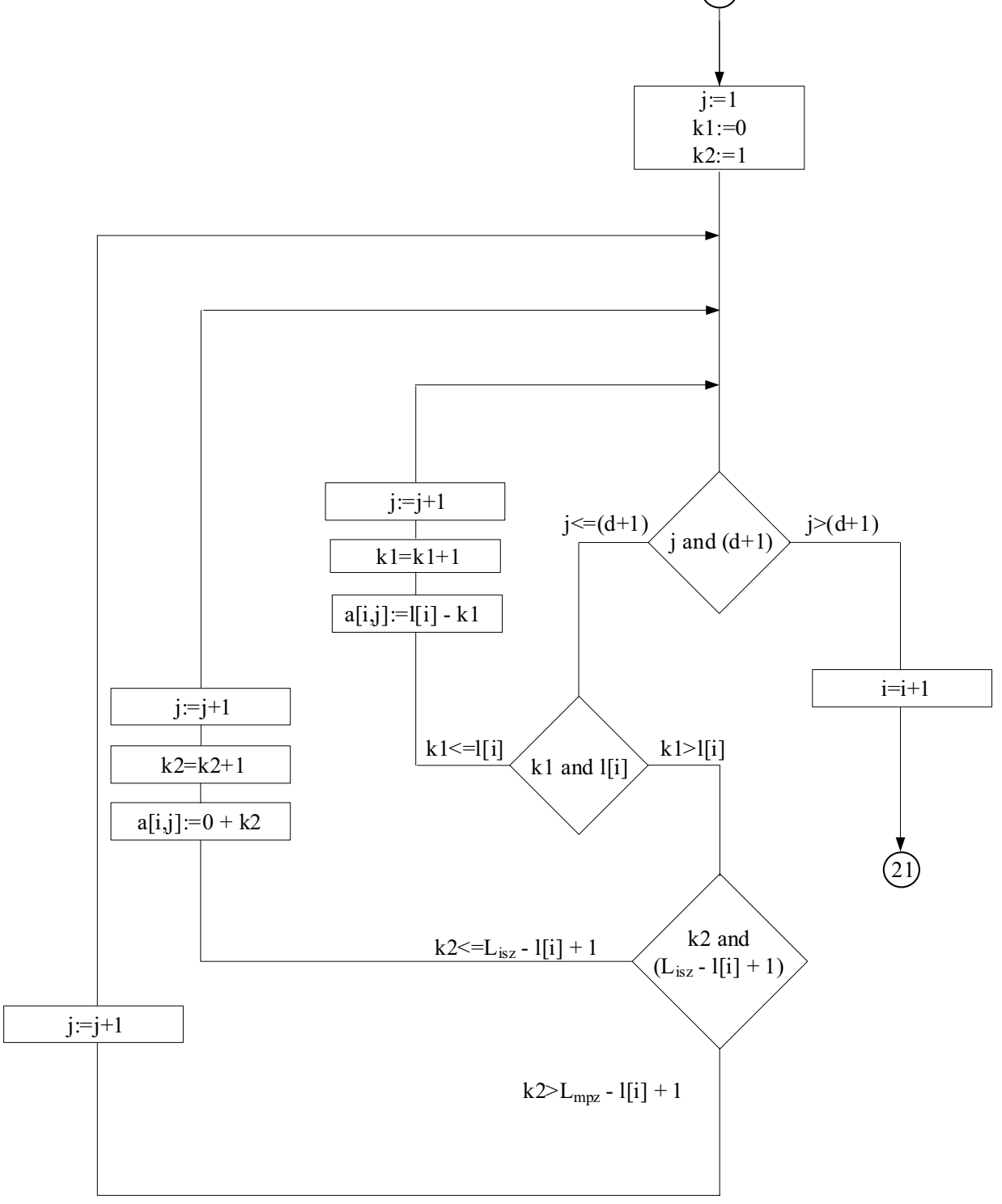

Fig. 6. Fragment №2 of the program operation algorithm. 


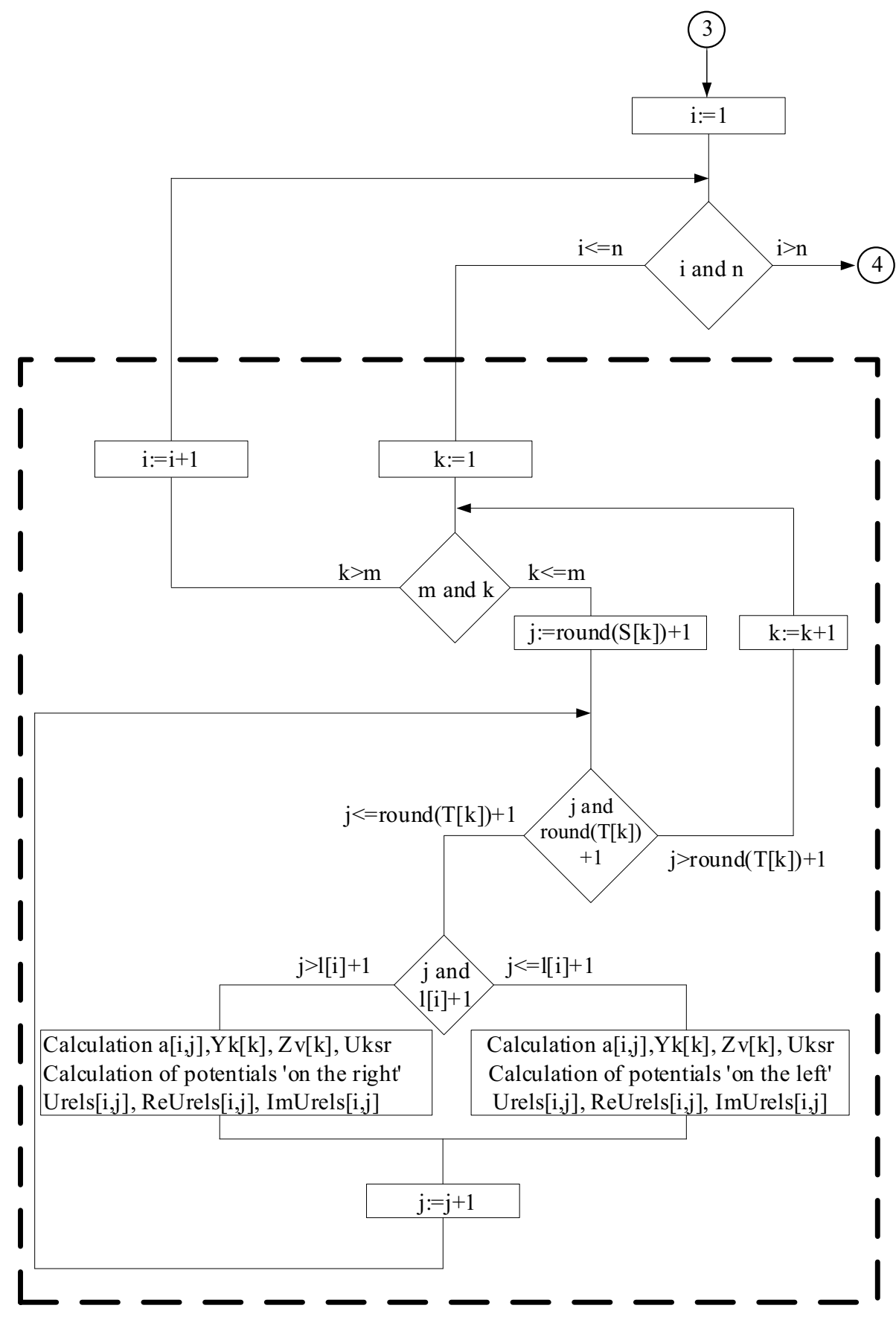

Fig. 7. Fragment №3 of the program operation algorithm. 


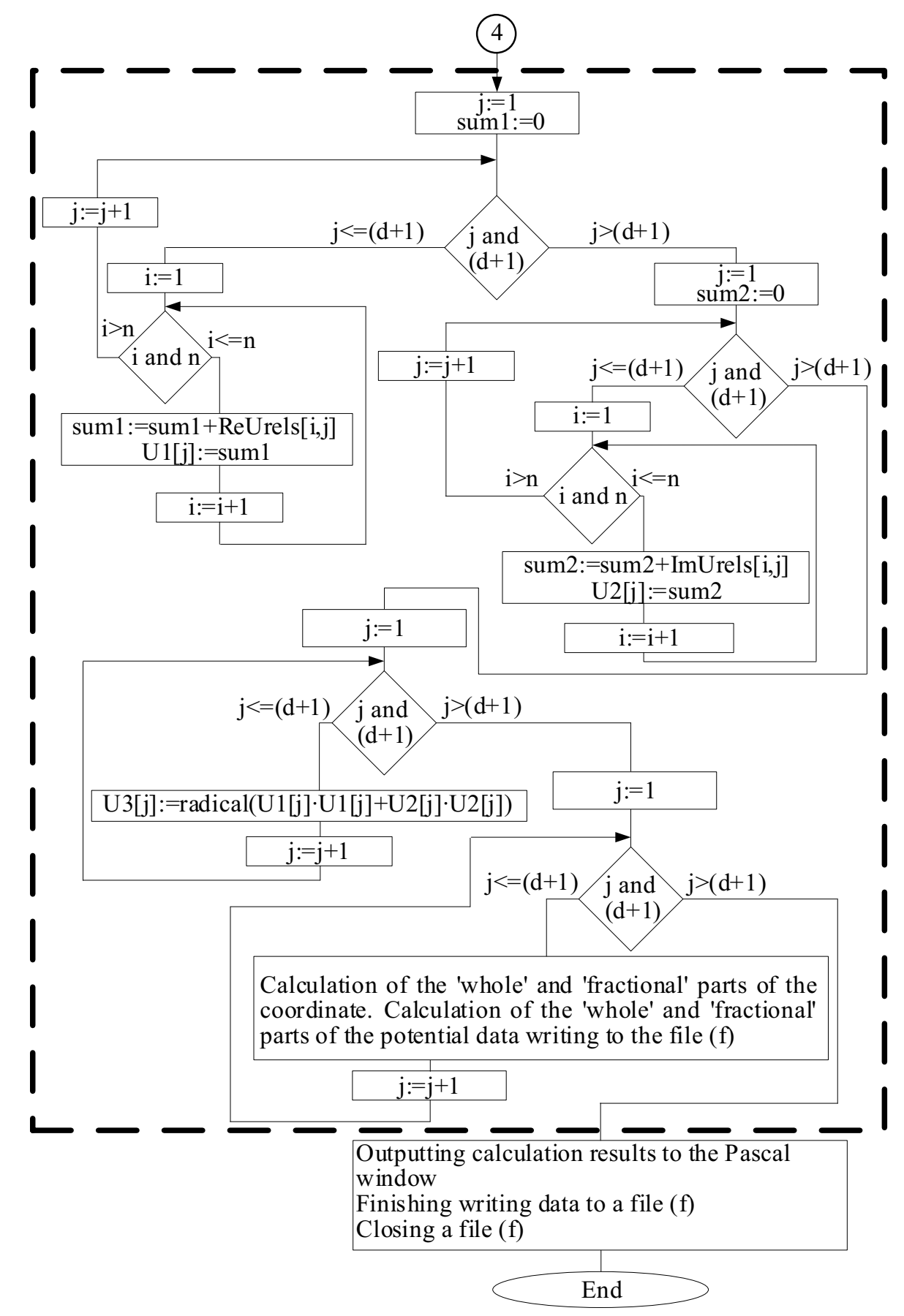

Fig. 8. Fragment №4of the program operation algorithm.

\section{Conclusions}

Upon the results of consideration of the issue of the rail-to-ground potentials growth in the reverse traction network, we can formulate the following conclusions: 
1. In addition to the traction current, the rail-to-ground potential value is largely influenced by the seasonally changing transient resistance, anti-spring measures taken, as well as the resistance of rails to alternating current of industrial frequency.

2. As a result of the research conducted, programs have been developed that allow calculating the distribution of rail-to-ground potentials within the inter-substation zone of any length.

3. Based on the algorithm obtained, developing calculation programs is necessary for a system with boasting transformers with a return conductor, as well as for a system with a shielding and amplifying conductor.

4. Based on the data obtained and real experiments, the calculation method should be adjusted to take into account all additional influencing factors.

\section{References}

1. V. Shamanov, Russ. Electr. Engin. 86, 548-549 (2015)

2. P. Colella, E. Pons, A. Tortora, AEIT International Annual Conference, 18346905 (2018)

3. V. Matta, G. Kumar, Electric Power Quality and Supply Reliability Conference (PQ), 303-308 (2014)

4. A. Mariscotti, IEEE Trans. Intell. Transp. Syst. 12, 201-210 (2011)

5. I. Ignatenko, E. Tryapkin, S. Vlasenko, A. Onischenko, V. Kovalev, Advances in Intelligent Systems and Computing 1115, 117-127 (2020)

6. R.W.B. Stell, IEEE Veh. Technol. Mag. 6, 12206592 (2011)

7. A. Ogunsola, A. Mariscotti,: Electromagnetic Compatibility in Railways (Springer Berlin Heidelberg, 2013)

8. P. Rui, W. Mingli, Y. Shaobing, International Conference on Sustainable Power Generation and Supply, SUPERGEN 2009, 11001154 (2009)

9. I. Ignatenko, S. Vlasenko, Advances in Intelligent Systems and Computing 1115, 6978 (2020)

10. I.V. Ignatenko, S.A. Vlasenko, Russ. Electr. Engin. 87(2), 65-67 (2016)

11. V. Shamanov, IEEE East-West Design \& Test Symposium (EWDTS), 18248723 (2018)

12. V. Li, L. Demina, S. Vlasenko, E3S Web Conf. 164, 03028 (2020)

13. I.V. Ignatenko, S.A. Vlasenko, IOP Conf. Ser.: Mater. Sci. Eng. 760, 012024 (2020)

14. I.V. Ignatenko, S.A. Vlasenko, IOP Conf. Ser.: Mater. Sci. Eng. 918, 012154 (2020)

15. S. Tetiana, K. Vitaliy, K. Yevheniia, IEEE 3rd International Conference on Intelligent Energy and Power Systems (IEPS), 18323445 (2018)

16. M. Mirzaei, P. Ripka, 53rd International Universities Power Engineering Conference (UPEC), 18319950 (2018)

17. V. Havryliuk, International Symposium on Electromagnetic Compatibility (EMC EUROPE), 251-254 (2018)

18. D. Bellan, G. Spadacini, F. Grassi, E. Fedeli, S.A. Pignari, Asia-Pacific Symposium on Electromagnetic Compatibility (APEMC), 15670514 (2013)

19. V.N. Li, L.S. Demina, S.A. Vlasenko, E.Y. Tryapkin, IOP Conf. Ser.: Mater. Sci. Eng. 918, $012118(2020)$ 
20. T. Serdiuk, M. Feliziani, K. Serdiuk, International Symposium on Electromagnetic Compatibility (EMC EUROPE), 18149852 (2018)

21. C. Charalambous, I. Cotton, P. Aylott, IEEE Trans. Power Deliv. 28, 13596015 (2013)

22. A. Baranovskii,: Autom. Control Comput. Sci. 45, 293-294 (2011)

23. A.N. Kobylitsky, I.V. Ignatenko, S.A. Vlasenko, E.Y. Tryapkin, I.A. Rebrov, IOP Conf. Ser.: Mater. Sci. Eng. 918 012185(2020) 